\title{
Genetic predisposition in children with cancer - affected families' acceptance of Trio-WES
}

\author{
Triantafyllia Brozou $^{1}$ • Julia Taeubner ${ }^{1} \cdot$ Eunike Velleuer $^{1} \cdot$ Martin Dugas $^{2}$ • \\ Dagmar Wieczorek $^{3}$ • Arndt Borkhardt ${ }^{1} \cdot$ Michaela Kuhlen $^{1}$ (D)
}

Received: 13 June 2017 /Revised: 10 August 2017 / Accepted: 13 August 2017 / Published online: 19 September 2017

(C) The Author(s) 2017, corrected publication October/2017. This article is an open access publication

\begin{abstract}
A considerable percentage of childhood cancers are due to cancer predisposition syndromes (CPS). The ratio of CPSs caused by inherited versus de novo germline mutations and the risk of recurrence in other children are unknown. We initiated a prospective study performing whole-exome se-
\end{abstract}

Arndt Borkhardt and Michaela Kuhlen contributed equally to this article.

Communicated by Peter de Winter

Electronic supplementary material The online version of this article (https://doi.org/10.1007/s00431-017-2997-6) contains supplementary material, which is available to authorized users.

Michaela Kuhlen

Michaela.Kuhlen@med.uni-duesseldorf.de

Triantafyllia Brozou

Triantafyllia.Brozou@med.uni-duesseldorf.de

Julia Taeubner

Julia.Taeubner@med.uni-duesseldorf.de

Eunike Velleuer

Eunike.Velleuer@med.uni-duesseldorf.de

Martin Dugas

Martin.Dugas@ukmuenster.de

Dagmar Wieczorek

Dagmar.Wieczorek@med.uni-duesseldorf.de

Arndt Borkhardt

Arndt.Borkhardt@med.uni-duesseldorf.de

1 Department of Pediatric Oncology, Hematology and Clinical Immunology, University Children's Hospital, Medical Faculty, Heinrich Heine University, Moorenstr. 5,

40225 Duesseldorf, Germany

2 Institute of Medical Informatics, University of Muenster, Muenster, Germany

3 Institute of Human Genetics, Medical Faculty, Heinrich Heine University, Duesseldorf, Germany quencing (WES) of parent-child trios in children newly diagnosed with cancer. We initially aimed to determine the interest in and acceptance of trio WES among affected families and to systematically collect demographic, medical, and family history data to analyze whether these point to an underlying CPS. Between January 2015 and December 2016, 83 (88.3\%) of 94 families participated; only $11(11.7 \%)$ refused to participate. Five $(6.0 \%)$ children presented with congenital malignancies and three $(3.6 \%)$ with tumors with a high likelihood of an underlying CPS. Two (2.5\%) families showed malignancies in family members $<18$ years, $11(13.8 \%)$ showed relatives $<45$ years with cancer, 37 (46.3\%) had a positive cancer history, and $14(17.5 \%)$ families had $>1$ relative with cancer.

Conclusions: Genetic testing in pediatric oncology is of great interest to the families, and the vast majority opts for investigation into potentially underlying CPSs. Trio sequencing provides unique insights into CPS in pediatric cancers and is increasingly becoming a common approach in modern oncology, and thus, trio sequencing needs also to be integrated routinely into the practice of pediatric oncology.

What is Known:

- A considerable percentage of childhood cancers are due to cancer predisposition syndromes (CPS).

What is New:

- Knowing about an underlying CPS and, thus, the risk of recurrence in other children is of great interest to affected families.

Keywords Cancer predisposition syndrome - Children . Trio $\cdot$ Whole-exome sequencing

Abbreviations

AACR Association of Cancer Research

ART Assisted reproductive technologies 


$\begin{array}{ll}\text { BWS } & \text { Beckwith-Wiedemann syndrome } \\ \text { CMMRD } & \text { Constitutional mismatch repair deficiency } \\ \text { CNS } & \text { Central nervous system } \\ \text { CPG } & \text { Cancer predisposition gene } \\ \text { CPS } & \text { Cancer predisposition syndrome } \\ \text { LFS } & \text { Li-Fraumeni syndrome } \\ \text { MAF } & \text { Minor allele frequency } \\ \text { NGS } & \text { Next generation sequencing } \\ \text { VUS } & \text { Variant of unknown significance } \\ \text { WES } & \text { Whole-exome sequencing }\end{array}$

\section{Introduction}

The proportion of children and adolescents with cancer attributable to an underlying cancer predisposition syndrome (CPS) is still unclear. Recent research studies indicate that a considerable percentage of childhood cancers are due to CPSs (16.7\% of non-central nervous system (CNS) solid tumors, $8.6 \%$ of CNS tumors, and $4.4 \%$ of leukemias) [20]. However, in the era of high-throughput sequencing, it might be supposed that new CPSs will be discovered and, thus, the identification of affected children and their families will presumably increase within the next decade [9]. In addition, the ratio of CPSs caused by inherited versus de novo germline mutations in cancer predisposition genes (CPGs) and, thus, the risk of recurrence in other children is almost completely unknown so far. For example, the number of inherited TP53 germline mutations causing Li-Fraumeni syndrome (LFS) is estimated to be as high as $75 \%$ [1].

Indeed, mutations in CPGs involved in the DNA repair machinery, including mismatch and double-strand break repair, might have immediate implications on clinical decisions. For instance, LFS patients are highly susceptible to radiationinduced tumorigenesis and alkylating chemotherapy and, thus, have an increased risk of developing secondary cancers $[5,7]$.

Whole-exome sequencing (WES) of parent-child trios has become a popular strategy to identify causative genetic variants in children with rare diseases [4, 10, 21]. However, it has not been routinely implemented in pediatric oncology as yet. A number of reports on children developing metachronous tumors and families with familial clustering of malignancies suggest that trio sequencing in pediatric oncology can identify underlying CPSs $[3,6,14]$.

Consequently, we initiated a monocentric prospective study on CPSs in a cohort of children and adolescents with a newly diagnosed malignancy by trio sequencing of the affected children and their parents. The main objectives of our study were first, to determine the interest in and acceptance of comprehensive clinical and molecular genetic evaluation in a pediatric oncology and hematology department; second, to systematically collect detailed demographic, medical, and family history data from this pediatric cancer cohort to analyze whether these data point to underlying CPSs; and third, to assess the proportion of children affected by either a wellknown or suspected underlying CPS including the distribution pattern of contributing CPGs.

\section{Patients and methods}

Since January 1st, 2015, an ongoing research study titled "Germline mutations in children with cancer" has been prospectively evaluating children and their parents by WES to test for underlying CPSs. All children (aged 0-18 years) with any newly diagnosed malignancy who were treated at the Department of Pediatric Oncology, Hematology and Clinical Immunology of the University Children's Hospital and their parents were eligible. Families whose children died before the informed consent process was completed were excluded from this analysis. No other exclusion criteria were defined.

\section{Informed consent process}

Informed consent was obtained in a multi-step process. In an initial conversation, the child (wherever possible) and the child's parents were informed about the diagnosis and the study by being provided with the study information. In a second step, a few days after the diagnosis, the family was notified about the study aims, benefits, and risks in more detail, including implications for the patient and the entire family, the possibility of incidental findings and variants of unknown significance (VUS), options and preferences regarding how results should be reported, and their "right not to know." In a third step, remaining questions were addressed and written informed consent was obtained. If the family was still undecided about participating, they were given more time for consideration. Pre-test counseling was provided by a pediatrician with a certificate in genetic counseling for genetic testing in pediatrics, as stipulated in the German gene diagnostic law. In cases where the family did not speak sufficient German, the informed consent process was performed with the help of a professional translator.

\section{Medical history and three-generation pedigree}

Demographic data and the child's medical history of previous malignancies and pre-existing conditions were collected through means of a standardized in-depth interview by a pediatrician (Supplement). This interview collected information regarding pregnancy, delivery, postnatal adaptation, development during early childhood, congenital anomalies, and other specific symptoms. As references for the comparison of birth data and data on assisted conception, the Annual Report (2016) of the Federal Statistical Office (Destatis) and the 
Annual Book (2015) of the German IVF Registry (DIR) were used.

All patients were thoroughly examined by a pediatrician, with particular attention to congenital anomalies and signs or conditions suggesting an underlying syndrome. Additionally, information on tumor/leukemia features pointing to an underlying germline defect was recorded and excessive toxicity to cancer therapy was prospectively evaluated.

Three-generation pedigrees (patient, parents, grandparents, siblings, uncles, and aunts) were constructed for each participating family, including information on birth date, deceased, age at and cause of death, symptoms.

DNA for WES analysis was extracted pre-therapeutically either from peripheral blood in patients with solid tumors or from skin biopsies using fibroblasts in patients with leukemia or lymphoma. Peripheral-blood-derived DNA from the parents was used for WES.

A bioinformatic pipeline was established based on data analysis published by the St. Judes study group and a constantly updated gene list currently comprising 2224 genes including the 565 known cancer-predisposing genes, which were summarized by Zhang et al. [20]. To identify only relevant single nucleotide variants (SNVs) by WES, we defined the following analysis criteria: (1) a high-quality DNA sequencing coverage of $\geq 250$-fold (to additionally identify parental mosaicism); (2) variants with a minor allele frequency (MAF) below 10\%; (3) SNVs in any of the 2224 genes of the cancer gene list with non-synonymous coding changes; (4) in silico prediction tools (SIFT and PolyPhen) considering the identified variant as (probably/possibly) damaging or deleterious for protein function; and (5) a CADD (combined annotation-dependent depletion) [8] score $>10$. An overview of the bioinformatic pipeline is given in Fig. 1 .

For this analysis, the data of patients enrolled between 1 January 2015, and 31 December 2016, were examined. The study was approved by the Ethics Committee of Heinrich Heine University, Duesseldorf, Germany (study number 4886).

\section{Results}

Between 1 January 2015, and 31 December 2016, 94 families of children and adolescents with a newly diagnosed malignancy were asked to participate in the study. Of these, 83 $(88.3 \%)$ families agreed to participate in the study and 11 $(11.7 \%)$ families refused to participate. Reasons for refusal were fear of the results in six cases (four of them with a positive cancer history in the family), uncertainty and mental overload in three families, and cultural objections in two families (none of them with a positive familial cancer history). In one of these cases, the adolescent patient refused to participate (due to fear) while both parents wanted to participate.

In 11 families, only one parent was available, due to either a lack of contact information (nine cases) or one parent having already died (two cases, both due to cancer). In one family, consent to participate in the study was given by both parents for the child but one parent (with a highly suspicious familial cancer history) refused to be tested him-/herself. Thus, in these cases, only duo sequencing was feasible. Details on recruitment and refusal are depicted in Fig. 2.

\section{Patient characteristics and medical history}

Demographic characteristics of participating families are listed in detail in Table 1. The mean number of children per family was 2.4 (range $1-6$ ).

In $80(96.4 \%)$ of 83 families, complete details on medical history including birth date, cause of death, and, in the case of a positive cancer history, also the type of cancer were available for further analysis in a three-generation pedigree. In addition to the information provided by the families, in some cases we also asked for and received medical records of the affected family members. Of these 80 children, three (3.8\%) children's parents reported the use of assisted reproductive technologies (ART), three (3.8\%) children presented with congenital heart defects, four $(5.0 \%)$ children with café-au-lait spots, six $(7.5 \%)$ children with pre-existing conditions (Asperger's syndrome, attention deficit hyperactivity disorder, depression, strabismus, splenic cyst, and hemangioma), and two (2.5\%) children with a history of a previous malignancy (further details are given in Table 2).

Comparing these data with data from the Federal Statistical Office and the German IVF Registry, no differences were observed in parental age, prematurity, or the number of reported ARTs used [16].

Of 83 children, most were diagnosed with leukemias (28, $33.7 \%$ ) and brain tumors $(19,22.9 \%)$ (Fig. 3a). Three (3.6\%) presented with tumors including hypodiploid ALL, plexus carcinoma, and pleuropulmonary blastoma (PPB) with a high likelihood of an underlying germline defect. Six (7.2\%) children developed excessive toxicity (grade 4 mucositis, neurotoxicity, veno-occlusive disease, hyperammonemia, and grade 5 respiratory failure) to cancer therapy, which was either particularly long-lasting or developed in a therapy regimen normally not associated with that kind of toxicity according to expert clinical experience. In two of these children, a CPS was subsequently diagnosed. Five $(6.0 \%)$ children presented with congenital leukemias or tumors (Table 3 ).

\section{Three-generation pedigree}

Three-generation pedigrees revealed malignancies in family members under the age of 18 years in two $(2.5 \%)$ of 80 
Fig. 1 Overview of the bioinformatic pipeline

\section{Alignment and variant calling}

$\rightarrow$ Alignment with BWA mem

$\rightarrow$ VarScan 2 trio SNV and indel calling

$\rightarrow$ VarScan2 false-positive filter

$\rightarrow$ SnpEff variant annotation and effect prediction

\section{Germline mutation filtration and annotation}

Gene list includes 2,224 genes:

$\rightarrow$ Cancer predisposing gene list (Zhang et al.)

$\rightarrow$ Cancer susceptibility and consenus list

$\rightarrow$ Gene list related to tumor entities

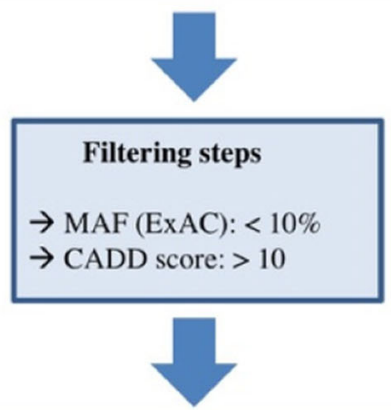

\section{Classification of SNVs and InDels into four groups}

1. nonsense, splice-site, frameshift variant in tumor suppressor genes

2. nonsense, splice-site, frameshift variant in non-tumor suppressor genes, predicted as damaging in dbSNPF by PolyPhen and Mutation Assessor

3. most likely benign, predicted as not damaging in dbSNFP

4. no annotation according to categories $1-3$

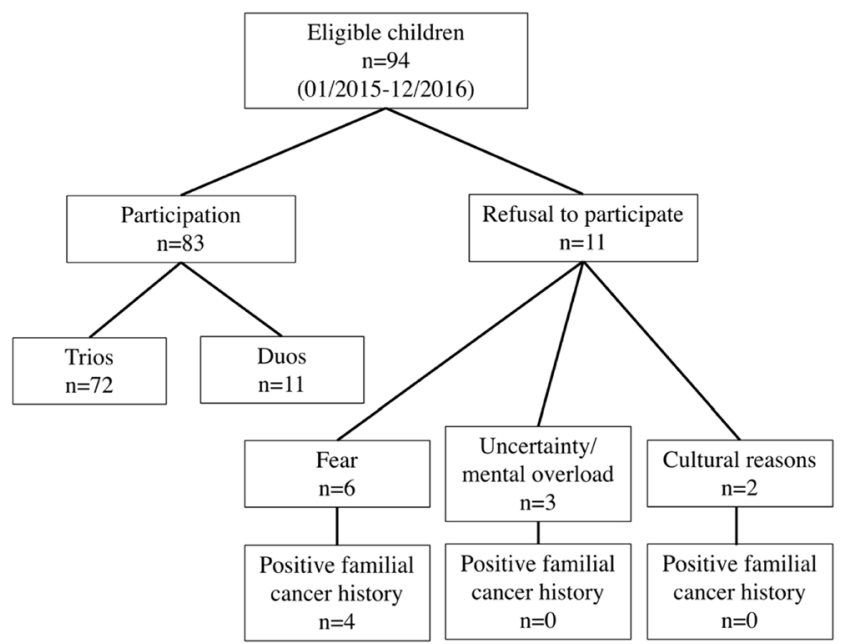

Fig. 2 Overview on participation and refusal reasons of families with children with a newly diagnosed malignancy $(n=94)$ families, relatives with cancer before the age of 45 years in 11 (13.8\%) families, any cancer history in 37 (46.3\%) families, and more than one relative with cancer in 14 (17.5\%) families

Table 1 Demographic characteristics of participating patients and their families $(n=83)$

\begin{tabular}{ll}
\hline Gender & \\
Male & $54(65.1 \%)$ \\
Female & $29(34.9 \%)$ \\
Age at onset in years, median (range) & 6.0 (birth-18.0 years) \\
Parental age in years, median (range) & \\
Father & $34.3(20.2-50.3)$ \\
Mother & $29.9(18.0-48.8)$ \\
Siblings, median (range) & \\
None & $23(27.7 \%)$ \\
$1-2$ & $47(56.6 \%)$ \\
$\geq 3$ & $13(15.7 \%)$ \\
\hline
\end{tabular}


Table 2 Details on medical history of the patients $(n=80)$

\begin{tabular}{|c|c|}
\hline \multicolumn{2}{|c|}{ Assisted reproductive technology } \\
\hline No & $76(95.0 \%)$ \\
\hline Hormonal & $1(1.3 \%)$ \\
\hline IVF/ICSI & $3(3.8 \%)$ \\
\hline \multicolumn{2}{|c|}{ Abnormalities during pregnancy } \\
\hline No & $62(77.5 \%)$ \\
\hline Yes & $18(22.5 \%)$ \\
\hline \multicolumn{2}{|c|}{ Small for gestational age } \\
\hline No & $75(93.8 \%)$ \\
\hline Yes & $5(6.3 \%)$ \\
\hline \multicolumn{2}{|l|}{ Prematurely born } \\
\hline No & $72(90.0 \%)$ \\
\hline Yes & $8(10.0 \%)$ \\
\hline \multicolumn{2}{|c|}{ Postpartal adaptation } \\
\hline Regular & $70(87.5 \%)$ \\
\hline Remarkable & $10(12.5 \%)$ \\
\hline \multicolumn{2}{|c|}{ Development in early childhood } \\
\hline Regular & $70(87.5 \%)$ \\
\hline Remarkable & $7(8.8 \%)$ \\
\hline Not applicable & $3(3.8 \%)$ \\
\hline \multicolumn{2}{|c|}{ Congenital anomalies } \\
\hline No & $73(91.3 \%)$ \\
\hline Yes & $7(8.8 \%)$ \\
\hline \multicolumn{2}{|c|}{$\begin{array}{l}\text { Pre-existing conditions other } \\
\text { than congenital anomalies }\end{array}$} \\
\hline No & $74(92.5 \%)$ \\
\hline Yes & $6(7.5 \%)$ \\
\hline \multicolumn{2}{|c|}{ History of previous malignancies } \\
\hline No & $78(97.5 \%)$ \\
\hline Yes & $2(2.5 \%)$ \\
\hline
\end{tabular}

(Table 4). No parents were consanguineous. More precisely, first- or second-degree relatives presented with (mostly premenopausal) breast cancer (including one father) in 16 $(20.0 \%)$ families, sarcoma in four $(5.0 \%)$ families, leukemia/ lymphoma in four $(5.0 \%)$ families, and colon cancer in ten $(12.5 \%)$ families. An overview of cancer diagnoses in first- or second-degree relatives is given in Fig. $3 \mathrm{~b}$.

\section{Reporting of results}

In case of an underlying CPS, validation of the identified mutation was carried out by Sanger sequencing before the results were reported to the families. To exclude sample swap, confirmation of the mutation was performed using a second peripheral blood sample by Sanger sequencing.

The treating pediatrician told the parents when the results were ready. If the parents wanted to know the results, a member of the study team met with the parents and, wherever possible, with the child to explain and discuss the results. In total, $82(98.8 \%)$ of 83 families wanted to be informed about

\section{a}

\section{- Leukemia}

Brain tumor

- Hodgkin lymphoma

non-Hodgkin lymphoma

- Neuroblastoma

Rhabdomyosarcoma

Bone tumor

Other

b

Breast cancer

- Sarcoma

- Leukemia/Lymphoma

Colon cancer

Liver cancer

- Skin cancer

Prostate cancer

- Lung cancer

Fig. 3 a Overview of the diagnoses of children with cancer enrolled in the study $(n=83)$. b Overview of cancer diagnoses in first- or seconddegree relatives. Same cancer entities were counted just once per family

the results, and none of them changed their minds when the results were available. Due to a positive family history and fear, one family did not want to be informed. When a CPS was identified, an appointment with a genetic counselor was recommended to the parents. If a CPS was then diagnosed, the affected child was integrated into a cancer surveillance program either according to published recommendations (for LFS) or individually conceptualized and subsequently adapted according to the recommendations of the Cancer Predisposition Workshop of the American Association of

Table 3 Tumor specifics and cancer therapy tolerance in participating children and adolescents $(n=83)$

\begin{tabular}{lc}
\hline Congenital tumor & \\
No & $78(94.0 \%)$ \\
Yes & $5(6.0 \%)$
\end{tabular}

Tumor with high likelihood of germline defect

$\begin{array}{lc}\text { No } & 79(95.2 \%) \\ \text { Yes } & 3(3.6 \%) \\ \text { Not applicable } & 1(1.2 \%)\end{array}$

Excessive toxicity to cancer therapy

$\begin{array}{lc}\text { No } & 74(89.2 \%) \\ \text { Yes } & 6(7.2 \%) \\ \text { Not applicable } & 3(3.6 \%)\end{array}$


Table 4 Details on threegeneration pedigree $(n=80)$

\begin{tabular}{lc}
\hline Malignancies in family members under the age of 18 years & $2(2.5 \%)$ \\
\hline Relatives with cancer $>18-45$ years of age & $11(13.8 \%)$ \\
$\geq 2$ first- or second-degree relatives in the same parental lineage with cancer under & $3(3.8 \%)$ \\
the age of 45 years & $37(46.3 \%)$ \\
Any cancer history & $14(17.5 \%)$ \\
More than 1 relative with cancer & $22(27.5 \%)$ \\
Deaths due to cancer & \\
\hline
\end{tabular}

Cancer research (AACR) $[11,12]$. Examples of children in whom diagnosis of a CPS led to adaptation of cancer therapy and/or inclusion in a cancer surveillance program, respectively, are given in Table 5.

As examples, brief descriptions of three families are given.

Case \#1: The 8-month-old boy was diagnosed with plexus carcinoma, a rare tumor with a high likelihood of an underlying germline defect. The parents did not have other children. The family history was highly suggestive of an existing CPS, including three second-degree relatives with osteosarcoma diagnosed under the age of 45 years and premenopausal breast cancer in the paternal lineage. However, genetic counseling had not been performed so far. Trio WES analysis confirmed a heterozygous germline mutation in TP53 (p. Gly245Ser, c. $733 \mathrm{G}>\mathrm{A}$ ), suggesting LFS, which is transmitted by the father and predicted to be pathogenic and disease causing. Response to international treatment recommendations (CPT SIOP 2009 including vincristine, cyclophosphamide, etoposide, doxorubicine, cisplatin, and actinomycin) was poor. Thus, normal radiotherapy was indicated. Due to an underlying LFS, an individual treatment concept omitting radiotherapy was created, which was based on drug resistance testing of the tumor (including bortezomib) and high-dose chemotherapy (including thiotepa, carboplatin, and etoposide) with autologous stem cell transplantation. On day +120 , the boy is well without evidence of relapse.

Case \#2: The 13-month-old girl presented with desmoplastic medulloblastoma and skin features reminiscent of constitutional mismatch repair deficiency (CMMRD). The non-consanguineous parents (both under the age of 30 years) and an older sister were healthy, as were three generations of the family. Two inherited homozygous VUS of MSH2 (c.274C>G, p.Leu92Val) and MSH6 (c.2426_2428delTAG, p.Val809del) were identified by WES and, thus, further raised the suspicion of CMMRD. As a differential diagnosis, germline mutations in POLD1 and POLE were ruled out [19]. Tumor microsatellite instability testing and immunohistochemistry analysis were inconclusive. Therefore, in collaboration with the CMMRD consortium [18], functional analyses were initiated to confirm diagnosis of CMMRD (Fremerey et al. submitted). The girl was treated according to the HIT guidance protocol without radiotherapy due to her young age. Two years onwards, the girl is well and in radiological complete remission.

Case \#3: The 10.5-year-old boy was diagnosed with periosteal osteosarcoma. His medical history was remarkable, with embryonal rhabdomyosarcoma of the thoracic wall at the age of 1.75 years. At that time, treatment comprised alkylating agents but no radiotherapy. His parents and dizygotic twin brother were healthy. An uncle in the paternal lineage was deceased due to cancer (further details were not available). Applying the abovementioned criteria for single nucleotide variants (SNVs) analysis, we did not detect any SNV fulfilling these conditions.

\section{Discussion}

Here, we evaluated the interest in and acceptance of comprehensive clinical and molecular genetic screening for an

Table 5 Examples of adaptation of cancer therapy and/or inclusion in a cancer surveillance program after diagnosis of a CPS by trio WES

\begin{tabular}{|c|c|c|}
\hline CPS and type of cancer & Adaptation of cancer therapy & $\begin{array}{l}\text { Cancer surveillance program } \\
\text { according to }\end{array}$ \\
\hline Li-Fraumeni syndrome in hypodiploid ALL & $\begin{array}{l}\text { Omission of cranial irradiation; instead, administration of } \\
\text { additional intrathecal chemotherapy }\end{array}$ & Kratz et al. Clin Canc Res (2017) \\
\hline Li-Fraumeni syndrome in plexus carcinoma & $\begin{array}{l}\text { Omission of cranial irradiation; instead, high-dose chemotherapy } \\
\text { with autologous stem cell transplantation }\end{array}$ & Kratz et al. Clin Canc Res (2017) \\
\hline $\begin{array}{l}\text { Constitutional mismatch repair deficiency in } \\
\text { medulloblastoma }\end{array}$ & None & Tabori et al. Clin Canc Res (2017) \\
\hline Dicer syndrome in pleuropulmonary blastoma & None & Schultz et al. Clin Canc Res (2017) \\
\hline Gorlin syndrome & None & Schultz et al. Clin Canc Res (2017) \\
\hline Neurofibromatosis type I in glioma & Omission of cranial irradiation; instead, systemic chemotherapy & Evans et al. Clin Canc Res (2017) \\
\hline
\end{tabular}


underlying CPS, including trio whole-exome sequencing of parents with a child diagnosed with cancer. Our data suggest that knowledge of an underlying CPS is of great interest to the families in our sample and that the vast majority of parents do not claim their right not to know. Instead, most families participated immediately, as they hoped to find a reasonable explanation for why their child had been struck with such an extraordinarily rare event in childhood and to learn about the risk of recurrence in their other children.

Whenever next generation sequencing (NGS) is initiated, according to the gene diagnostic law, the treating physician is obligated to discuss the full range of benefits, risks, and alternatives of this particular genetic test including the potential to reveal gene abnormalities related to other disorders. However, disclosing the diagnosis of cancer is overwhelming and dramatically limits the child's/parents' receptivity. Consequently, in the daily clinical routine of pediatric oncology, this leads to the imperative necessity for a time-consuming multi-step process as depicted above. Indeed, the decision-making process of the families sometimes takes a few months. In our sample, only a minority of families exercised their "right not to know". These were either families with a highly suspicious familial cancer history or families who refused to participate due to their cultural background. This is in line with the empirical expert knowledge that parents frequently ask whether their other children have an increased risk of developing cancer.

Identifying children with a hereditary CPS by trio WES has far-reaching consequences that extend beyond providing cancer care for the child. Close and more distant relatives might likewise be affected despite being young and as-yet asymptomatic. Disclosing a hereditary CPS in these relatives might be clinically relevant and even lifesaving on the one hand, as it provides the excellent possibility to initiate early cancer surveillance programs [11]. On the other hand, it constitutes an enormous life-long burden of knowledge and might deeply affect quality of life and family planning. In this context, the potential advantages and drawbacks as well as personal autonomy regarding the "right not to know" must be discussed in detail before initiating trio WES. An appointment with a genetic counselor was strongly recommended to families in which an underlying CPS was diagnosed. However, a discussion about further genetic testing of additional family members must consider that, in contrast to standards for genetic testing in adults, predictive testing in children is recommended only when the disease is associated with childhood onset, and only with available effective screening and/or intervention options [2, 13]. Refraining from predictive testing in childhood allows the child to make this decision autonomously when reaching adulthood. As one child had already developed cancer, disease onset during childhood is given in all families. Nevertheless, cancer surveillance programs which are advantageous to survival exist only for LFS to date, but still need to be established and proved to be beneficial for other CPS.
We could not identify features in pregnancy, delivery, congenital anomalies, postnatal adaptation, or development during early childhood that pointed towards an underlying CPS. Moreover, neither parental age nor ART seems to be associated with an increased cancer risk in our study cohort. This is in line with previous findings that children born after ART are not at increased cancer risk [15, 17].

However, $3-8 \%$ of the children presented with suggestive clinical features (e.g., café-au-lait spots), tumors with a high likelihood of an underlying germline defect, or excessive toxicity to cancer therapy. In addition, in a remarkable number of families, the three-generation pedigree revealed a highly suggestive family history. Thus, although this is an observational study with respective limitations, our preliminary findings demonstrate that a thorough clinical examination and indepth family history might point towards an underlying CPS, which is in contrast to previous findings by Zhang et al. [20].

However, a highly suggestive medical history, such as one including metachronous tumors, or an unremarkable family history could both be misleading. The latter is of particular importance, as the number of de novo TP53 germline mutations causing LFS is estimated to be as high as $25 \%$ [1]. Notably, the proportion of CPSs caused by de novo germline mutations in other CPGs is so far completely unknown.

Thus, our study provides a highly valuable resource to determine the type, frequency, and the de novo mutation rate of CPSs in a cohort of newly diagnosed pediatric cancer patients and may eventually identify novel CPSs in the future.

\section{Conclusions}

In pediatric oncology, testing for an underlying CPS seems to be more important to the affected families than exercising their right of not knowing. In order to gain better insights into the ratio between inherited risk alleles found throughout the family and acquired de novo mutations, trio sequencing needs to be integrated routinely into the practice of pediatric oncology

Acknowledgements This work was supported by the Elterninitiative Kinderkrebsklinik e.V. Duesseldorf. The authors thank Dr. S. Balzer and Dr. C. Potthoff for their contributions in sample acquisition, Dr. M. Remke for drug resistance testing, Dr. M. Gombert for running the next generation sequencing analysis, M.Sc. S. Ginzel for the internal SQL database, Mrs. S. Furlan, Mrs. K. Alemazkour, and Mrs. S. Stuhlsatz for technical assistance, and Dr. J.I. Hoell for critical reading of the manuscript. Parts of this work were performed as essential parts of the doctoral thesis of TB.

Authors' contributions TB collected the data and first drafted the manuscript. JT assisted in data management, was responsible for sample management, and revised the manuscript for important intellectual content. EV helped in collecting the data and assisted in data management. MD contributed to the WES analysis. DW contributed to genetic 
counseling. AB designed and supervised the project and critically reviewed and revised the manuscript for important intellectual content. MK designed and supervised the project, supervised data collection and analysis, and first drafted the manuscript. All authors approved the final manuscript as submitted and agree to be accountable for all aspects of the work.

Funding This work was supported by the Elterninitiative Kinderkrebsklinik e.V. Duesseldorf.

Compliance with ethical standards The study was approved by the Ethics Committee of Heinrich Heine University, Duesseldorf, Germany (study number 4886).

Conflict of interest The authors declare that they have no conflict of interest.

Informed consent Informed consent was obtained from the parents and legal guardians respectively.

Open Access This article is distributed under the terms of the Creative Commons Attribution 4.0 International License (http:// creativecommons.org/licenses/by/4.0/), which permits unrestricted use, distribution, and reproduction in any medium, provided you give appropriate credit to the original author(s) and the source, provide a link to the Creative Commons license, and indicate if changes were made.

\section{References}

1. Chompret A, Brugieres L, Ronsin M, Gardes M, Dessarps-Freichey F, Abel A, Hua D, Ligot L, Dondon MG, Bressac-de Paillerets B, Frebourg T, Lemerle J, Bonaiti-Pellie C, Feunteun J (2000) P53 germline mutations in childhood cancers and cancer risk for carrier individuals. Br J Cancer 82:1932-1937

2. Clayton EW, McCullough LB, Biesecker LG, Joffe S, Ross LF, Wolf SM; Clinical Sequencing Exploratory Research Consortium Pediatrics Working G (2014) Addressing the ethical challenges in genetic testing and sequencing of children. Am J Bioeth 14:3-9

3. Fremerey J, Balzer S, Brozou T, Schaper J, Borkhardt A, Kuhlen M (2016) Embryonal rhabdomyosarcoma in a patient with a heterozygous frameshift variant in the DICER1 gene and additional manifestations of the DICER1 syndrome. Fam Cancer 16:401-405

4. Ghosh S, Honscheid A, Duckers G, Ginzel S, Gohlke H, Gombert M, Kempkes B, Klapper W, Kuhlen M, Laws HJ, Linka RM, Meisel R, Mielke C, Niehues T, Schindler D, Schneider D, Schuster FR, Speckmann C, Borkhardt A (2016) Human RAD52: a novel player in DNA repair in cancer and immunodeficiency. Haematologica 102:e69-e72

5. Heymann S, Delaloge S, Rahal A, Caron O, Frebourg T, Barreau L, Pachet C, Mathieu MC, Marsiglia H, Bourgier C (2010) Radioinduced malignancies after breast cancer postoperative radiotherapy in patients with Li-Fraumeni syndrome. Radiat Oncol 5:104

6. Hoell JI, Gombert M, Ginzel S, Loth S, Landgraf P, Kafer V, Streiter M, Prokop A, Weiss M, Thiele R, Borkhardt A (2014) Constitutional mismatch repair-deficiency and whole-exome sequencing as the means of the rapid detection of the causative MSH6 defect. Klin Padiatr 226:357-361

7. Kappel S, Janschek E, Wolf B, Rudas M, Teleky B, Jakesz R, Kandioler D (2015) TP53 germline mutation may affect response to anticancer treatments: analysis of an intensively treated LiFraumeni family. Breast Cancer Res Treat 151:671-678

8. Kircher M, Witten DM, Jain P, O'Roak BJ, Cooper GM, Shendure J (2014) A general framework for estimating the relative pathogenicity of human genetic variants. Nat Genet 46:310-315

9. Kuhlen M, Borkhardt A (2015) Cancer susceptibility syndromes in children in the area of broad clinical use of massive parallel sequencing. Eur J Pediatr 174:987-997

10. Kuhlen M, Honscheid A, Loizou L, Nabhani S, Fischer U, Stepensky P, Schaper J, Klapper W, Siepermann M, Schuster F, Meisel R, Borkhardt A (2016) De novo PIK3R1 gain-of-function with recurrent sinopulmonary infections, long-lasting chronic CMV-lymphadenitis and microcephaly. Clin Immunol 162:27-30

11. Raskind WH, Hisama FM, Bennett RL (2016) Biochemical and imaging surveillance in Li-Fraumeni syndrome. Lancet Oncol 17: e472

12. Ripperger T, Bielack SS, Borkhardt A, Brecht IB, Burkhardt B, Calaminus G, Debatin KM et al (2017) Childhood cancer predisposition syndromes - a concise review and recommendations by the Cancer Predisposition Working Group of the Society for Pediatric Oncology and Hematology. Am J Med Genet A 173: 1017-1037

13. Ross LF, Saal HM, David KL, Anderson RR; American Academy of P, American College of Medical G, Genomics (2013) Technical report: ethical and policy issues in genetic testing and screening of children. Genet Med 15:234-245

14. Samuel N, Villani A, Fernandez CV, Malkin D (2014) Management of familial cancer: sequencing, surveillance and society. Nat Rev Clin Oncol 11:723-731

15. Sundh KJ, Henningsen AK, Kallen K, Bergh C, Romundstad LB, Gissler M, Pinborg A, Skjaerven R, Tiitinen A, Vassard D, Lannering B, Wennerholm UB (2014) Cancer in children and young adults born after assisted reproductive technology: a Nordic cohort study from the Committee of Nordic ART and Safety (CoNARTaS). Hum Reprod 29:2050-2057

16. Wieczorek D, Ludwig M, Boehringer S, Jongbloet PH, GillessenKaesbach G, Horsthemke B (2007) Reproduction abnormalities and twin pregnancies in parents of sporadic patients with oculoauriculo-vertebral spectrum/Goldenhar syndrome. Hum Genet 121:369-376

17. Williams CL, Bunch KJ, Stiller CA, Murphy MF, Botting BJ, Wallace WH, Davies M, Sutcliffe AG (2013) Cancer risk among children born after assisted conception. N Engl J Med 369:18191827

18. Wimmer K, Kratz CP, Vasen HF, Caron O, Colas C, Entz-Werle N, Gerdes AM, Goldberg Y, Ilencikova D, Muleris M, Duval A, Lavoine N, Ruiz-Ponte C, Slave I, Burkhardt B, Brugieres L, CMMRD EU-CCf(2014) Diagnostic criteria for constitutional mismatch repair deficiency syndrome: suggestions of the European consortium 'care for CMMRD' (C4CMMRD). J Med Genet 51: 355-365

19. Wimmer K, Beilken A, Nustede R, Ripperger T, Lamottke B, Ure B, Steinmann D, Reineke-Plaass T, Lehmann U, Zschocke J, Valle L, Fauth C, Kratz CP (2017) A novel germline POLE mutation causes an early onset cancer prone syndrome mimicking constitutional mismatch repair deficiency. Fam Cancer 16:67-71

20. Zhang J, Walsh MF, Wu G, Edmonson MN, Gruber TA, Easton J, Hedges D et al (2015) Germline mutations in predisposition genes in pediatric cancer. N Engl J Med 373:2336-2346

21. Zhu X, Petrovski S, Xie P, Ruzzo EK, Lu YF, McSweeney KM, Ben-Zeev B et al (2015) Whole-exome sequencing in undiagnosed genetic diseases: interpreting 119 trios. Genet Med 17:774-781 